\title{
Protocolo de exercícios terapêuticos em grupo para pessoas com doença de Alzheimer
}

\section{Protocol of group therapeutic exercises of people with Alzheimer's Disease}

\author{
Carolina Quirino Dias ${ }^{1}$ (1) \\ João Antonio de Sousa Barros 2 (1) \\ Zodja Graciani ${ }^{3}$ (1) \\ Cibelle Albuquerque de la Higuera Amato 4 (1) \\ Étria Rodrigues 5 \\ Denise Loureiro Vianna ${ }^{6}$ (1) \\ Susi Mary de Souza Fernandes 7 (1)
}

\author{
1'Autora para correspondência. Universidade Presbiteriana Mackenzie (São Paulo). São Paulo, Brasil. carol.quirinodias@gmail.com \\ 2Universidade de São Paulo (São Paulo). São Paulo, Brasil. joaobarros@usp.br \\ 3.7Universidade Presbiteriana Mackenzie (São Paulo). São Paulo, Brasil. zodja.graciani@mackenzie.br, cibelle.amato@mackenzie.br, \\ etria.rodrigues@mackenzie.br, denise.vianna@mackenzie.br, susimary.fernandes@mackenzie.br
}

RESUMO | INTRODUÇÃO: O envelhecimento gera diversas alterações biológicas que dão origens a condições de saúde como a Doença de Alzheimer (DA) que pode prejudicar a funcionalidade dos idosos. OBJETIVO: Verificar o efeito de um programa de exercícios fisioterapêuticos sobre a saúde dos idosos com DA. MÉTODos: Foi realizado um estudo longitudinal, em idosos com DA frequentadores de um Centro-Dia. Os participantes foram selecionados por meio de características demográficos e condições de saúde. Para análise das variáveis de desfecho foram avaliados pré e pós intervenção com os testes: Timed Up and Go (TUG), Caixa e Blocos, Escala de Berg e Alcance funcional em pé e sentado. Em seguida foram submetidos a um protocolo de intervenção em grupo dividido em três fases: fase preparatória, fase ativa e fase de desaquecimento. Utilizou-se para análise estatística o Teste T-Student Pareado e a correlação de Pearson. Foi estabelecido Intervalo de Confiança (IC) e $p<0,05$. RESULTADOS: Foram realizadas 28 sessões com 11 idosas, com idade média de $88( \pm 4)$ anos, frequência semanal no Centro Dia de $4( \pm 1)$ vezes e pontuação média no MEEM de $12( \pm 5)$. Obtiveram significância estatística no teste Alcance Sentado pré $22,3( \pm 10,4)$ e pós $28,2( \pm 9,0)$, com $p=0,003$ e, no teste de TUG pré $16,9( \pm 5,8)$ e pós $2,5( \pm 3,9)$, com $p=0,009$. Além disso, o teste Berg e TUG apresentaram correlação negativa, classificada como boa $p=$ 0,020. CONCLUSÃO: O protocolo proposto contribuiu para melhora na saúde funcional observadas no aumento do alcance funcional e da mobilidade das idosas.

PALAVRAS-CHAVE: Doença de Alzheimer. Modalidades de fisioterapia. Exercício terapêutico. Idoso.
ABSTRACT | INTRODUCTION: Aging generates several biological changes that give rise to diseases such as Alzheimer's Disease (AD) that can result in the functionality of the elderly. OBJECTIVE: To verify the effect of a physical therapy exercise program on the health of elderly people with AD. MATERIALS AND METHODS: A longitudinal study was carried out in elderly people with AD who attended a Day Care Center. Participants were selected using demographic characteristics and health conditions. For the analysis of the outcome variables, the pre and post intervention were evaluated with the tests: Timed Up and Go (TUG), Box and Blocks, Berg Scale and Functional reach in standing and sitting. Then they underwent a group intervention protocol divided into three phases: the preparatory phase, the active phase and the cooling down phase. Paired T-Student Test and Pearson's correlation were used for statistical analysis. Confidence Interval $(\mathrm{Cl})$ was established and $p<0.05$. RESULTS: We performed 28 sessions, with 11 elderly women, mean age of $88( \pm 4)$ years, weekly attendance at the Center on Day $4( \pm 1)$ times and mean score on the MMSE of $12( \pm 5)$. They obtained statistical significance in the Seated Reach test pre 22.3 ( \pm 10.4 ) and post $28.2( \pm 9.0)$, with $p=0.003$ and, in the TUG test pre 16.9 $( \pm 5.8)$ and powders $2.5( \pm 3.9)$, with $p=0.009$. In addition, the Berg and TUG test showed a negative correlation, classified as good $p=0.020$. CONCLUSION: The proposed protocol contributed to the improvement in functional health observed in the increase in the functional range and mobility of the elderly.

KEYWORDS: Alzheimer Disease. Physical therapy modalities. Exercise therapy. Aged. 


\section{Introdução}

O envelhecimento é um processo dinâmico e progressivo marcado por modificações morfológicas, funcionais, bioquímicas e psicológicas que resultam em perda das capacidades de adaptação do indivíduo ao meio ambiente - senescência. O que oferece uma maior vulnerabilidade, diminuição da capacidade funcional e, consequentemente maior incidência de adoecimento - senilidade ${ }^{1}$ Com o fenômeno da transição demográfica estima-se que em 2050 haverá 253 milhões de idosos no mundo e uma expectativa de vida de 88 anos $^{2}$. Tal fato fez crescer a preocupação em relação à manutenção da capacidade funcional nessa população 3 .

Uma das alterações biológicas é a degeneração do sistema nervoso central dando origem às Demências. A mais comum é a doença de Alzheimer (DA), com prevalência aumentada de $0,7 \%$ dos 60 a 64 anos de idade para cerca de $40 \%$ nos grupos etários de 90 a 95 anos$^{4}$. Caracterizada pela degeneração sináptica, perda neuronal e acúmulo no córtex de placas senil, e emaranhado neurofibrilares. Tem um início insidioso $^{5}$ com manifestação cognitiva leve associada a alterações na coordenação motora grossa. Nos estágios mais avançados o comprometimento cognitivo e motor é agravado com redução significativa na capacidade funcional $\underline{\underline{6}}$.

Pesquisas têm evidenciado resultados benéficos com a prática regular da atividade física nas funções cognitivas e na capacidade funcional em idosos com demência ${ }^{7}$. De forma direta aumenta a velocidade do processamento cognitivo por constituírem uma melhora da circulação cerebral e alterações na síntese e degradação de neurotransmissores promovendo mudanças em estruturas cerebrais que meIhoram a agilidade, o equilíbrio e o desempenho do padrão da marcha4.

Com a necessidade de proporcionar melhor qualidade de vida aos idosos com DA criou-se os Centros-Dia, cujo objetivo é ofertar, durante o dia, cuidados pessoais como forma de suplementar os cuidados familiares. Nesse sentido, em 2005, foi criada a Associação dos Familiares e Amigos dos Idosos (AFAl). Uma instituição sem fins lucrativos, com um olhar integrador social, saúde e bem-estar sobre a família e sobre o idoso com DA por meio de assistência multiprofissional.
Diante do novo cenário demográfico e as repercussões sobre o cuidado com a pessoa idosa com DA surgiu a pergunta: Idosos com DA submetidos a um protocolo fisioterapêutico tem melhora na saúde funcional? Para responder a pergunta do estudo tornou-se relevante elaborar e aplicar um protocolo de exercícios fisioterapêuticos sobre a saúde funcional dos idosos com DA.

\section{Métodos}

\section{Aspectos Éticos}

O projeto foi submetido a Plataforma Brasil, CAAE: 92458518.4.0000.0084 e aprovado sob número 2.886.421. Em seguida foi realizado o contato com a instituição parceira e com todos os responsáveis pelos participantes selecionados de acordo com os objetivos da pesquisa. Estes receberam todas as informações necessárias e obtiveram as dúvidas esclarecidas e, a participação foi confirmada por meio da coleta das assinaturas dos Termos de consentimento livre e esclarecido (TCLEs). Após o consentimento dos responsáveis, os idosos foram consultados por meio do Termo de Assentimento (TA) e aqueles que consentiram em participar foram iniciadas as coletas de dados.

\section{Alocação dos Participantes}

Os participantes são frequentadores de um CentroDia da cidade de São Paulo, parceiro da Universidade sede da pesquisa.

Foram incluídos no estudo idosos com 60 ou mais anos de idade, de ambos os sexos, com diagnóstico médico de DA leve e moderada com capacidade cognitiva que permita compreensão das tarefas, que frequentam o Centro-Dia, no mínimo 2x por semana, sem comorbidades associadas que comprometam a mobilidade.

\section{Procedimentos}

Foi realizado um estudo longitudinal de intervenção por meio de um protocolo de exercício fisioterapêutico. 
A primeira parte do estudo consistiu na seleção dos participantes, por análise dos prontuários e avaliação do estado mental. Os dados coletados foram utilizados para caracterização da mostra foram: a) confirmação de diagnóstico médico de DA; b) características demográficas (sexo, idade, data de nascimento, escolaridade, frequência semanal, condições de saúde e independência funcional); c) perfil nutricional dos idosos (peso, altura, IMC, medidas das circunferências). Os idosos selecionados foram submetidos à avaliação do estado mental pela escala Mini-Exame do Estado Mental (MEEM).

O MEEM é composto por diversas questões agrupadas em 7 categorias, cada uma delas com o objetivo de avaliar "funções" cognitivas específicas: orientação para tempo, orientação para local, registro de 3 palavras, atenção e cálculo, lembrança das 3 palavras, linguagem, e capacidade construtiva visual. O escore pode variar entre 0 e 30 pontos, sendo pontuação igual ou superior a 27 sem alteração cognitiva, pontuação de 21 a 24 pontos perda cognitiva leve, pontuação de 20 a 10 perda cognitiva moderada e pontuação igual ou menor que 09 perda cognitiva grave ${ }^{8}$.

Para análise das variáveis de desfecho os participantes foram submetidos a avaliações pré e pós-intervenções em um único dia que consistiu em:

i. Avaliação da função psicomotora que incluiu tempo de reação, tempo de movimento e velocidade do desempenho e, para tanto foram utilizados os testes Timed Up and Go (TUG) e Caixa e Blocos.

O TUG foi utilizado para avaliação da mobilidade funcional na qual a velocidade do desempenho foi anotada. O idoso foi orientado a levantar-se de uma cadeira com encosto, caminhar três metros, dar a volta, retornar a cadeira e sentar com as costas apoiadas no encosto. O tempo para realização da tarefa foi cronometrado. A realização do teste em até 10 segundos é considerada normal para indivíduos independentes e sem risco de quedas, entre 11 e 20 segundos é o esperado para idosos frágeis, com independência parcial e com baixo risco de quedas, acima de 20 segundos indica déficit importante da mobilidade física e risco de quedas?.
O teste com a Caixa de blocos (Box of Blocks) avaliou a destreza manual por meio da análise do tempo de reação, movimento e velocidade do desempenho. $\mathrm{O}$ teste foi realizado em um ambiente bem iluminado, com idoso sentado em uma cadeira adequada para seu tamanho, com a caixa posicionada a sua frente em posição horizontal. O idoso é orientado a passar um bloco de cada vez para o outro compartimento da caixa em 1 minuto primeiro com a mão dominante e depois com a mão não dominante. $O$ resultado foi avaliado de acordo com a quantidade de blocos que o idoso conseguiu passar em 1 minuto ${ }^{10}$.

ii. Avaliação do desempenho motor, força muscular e flexibilidade por meio da Escala de Berg, e alcance funcional em pé e sentado.

A Escala de Equilíbrio de Berg (EEB) foi empregada para avaliar o equilíbrio e o risco de quedas em idosos. Foram executadas 14 tarefas, as quais foram classificadas de 0 a 4, sendo que 0 representa a incapacidade e 4 capacidade total para a realização da tarefa. As tarefas são: 1) Transferência da posição sentada para posição em pé; 2) Permanecer em pé sem apoio; 3) Permanecer sentado sem apoio; 4) Transferência da posição em pé para posição sentada; 5) Transferências; 6) Permanecer em pé com os olhos fechados; 7) Permanecer em pé com os pés juntos; 8) Alcançar a frente com os braços estendidos; 9) Pegar um objeto do chão; 10) Virar-se para olhar para trás; 11) Girar 360 graus; 12) Posicionar os pés alternadamente no degrau; 13) Permanecer em pé com um pé à frente; 14) Permanecer em pé sobre um pé11.

O escore total da EEB é de 56 pontos, sendo pontuação de 56 a 41 classificado como baixo risco de queda, pontuação de 40 a 21 classificado como médio risco de queda e pontuação de 20 a 0 classificado como alto risco de queda ${ }^{11}$.

O Teste de Alcance Funcional Anterior (TAF) foi realizado com uma fita métrica presa a parede, paralela ao chão, posicionada na altura do acrômio do idoso. O idoso foi posicionado com os pés paralelos entre si, perpendicular em relação à parede e próximo ao início da fita métrica. Com punhos em posição neutra, cotovelos estendidos e ombro com flexão de $90^{\circ}$, o idoso foi orientado a realizar a inclinação para 
frente sem tocar na fita e, em seguida foi verificado o deslocamento sobre ela. O resultado do teste foi representado pela média, após três tentativas, da diferença entre a medida na posição inicial e a final registrada na régua. Deslocamentos menores que 15 centímetros indicam fragilidade do indivíduo e risco de queda; 16 a $25 \mathrm{~cm}$ indica baixo risco de quedas e acima de $25 \mathrm{~cm}$ indica sem risco de queda ${ }^{9}$.

E o teste de Alcance Funcional Anterior sentado que tem o mesmo princípio, porém o idoso foi posicionado em um banco sem encosto, com altura adequada para que mantenha os pés apoiados no chão, com ombro fletido a $90^{\circ}$ e cotovelo estendido. Ao inclinarse para frente o máximo possível sem encostar à parede e sem levantar as nádegas do banco ${ }^{12}$.

A partir da avaliação inicial os participantes foram submetidos a um protocolo de intervenção em grupo, realizado $2 x$ semanais, de terças e quintas-feiras, das 8 horas as 11 horas, com duração de 10 semanas.

A intervenção foi dividida em três fases: fase preparatória com duração de 15 minutos; fase ativa com duração de 2 horas subdividida respectivamente em circuito funcional (50 minutos), pausa (10 minutos), caminhada (20 minutos), pausa (10 minutos) e exercícios terapêuticos (30 minutos) e; fase de desaquecimento com duração de 10 minutos.

A fase preparatória continha exercícios de alongamento de cabeça, membros superiores (MMSS) e membros inferiores (MMII) com duração de 30 segundos para cada movimento; exercícios de mobilidade e flexibilidade articular de membro superior com utilização de pano terapêutico e exercícios respiratórios associados à flexão de MMSS todos com 3 séries de 10 repetições.

A fase ativa iniciava com circuito funcional com estações de tarefas que envolviam equilíbrio, alcance funcional, coordenação motora global e fina e, duplas tarefas para cognição, cada estação com duração de 3 minutos. Após a realização desta etapa os idosos eram instruídos a sentar, hidratar-se e realizar lanche da manhã, com dieta estabelecida pela nutricionista do local.
Em seguida, os idosos saiam para a caminhada acompanhados pelas cuidadoras do local, a caminhada consistia em dois percursos. Um considerado curto indicado para idosos dependentes e, um longo para idosos com maior independência. Ao retornar os idosos eram instruídos para hidratação e as variáveis fisiológicas eram coletadas como meio de controle do estado geral.

Após esta etapa eram realizados os exercícios terapêuticos de mobilidade de tronco e MMSS utilizando bastões 3 séries de 10 repetições, força muscular de MMSS e MMII com foco em aumentar potência muscular (baixa carga, pouca repetição e velocidade rápido) $)^{13}$ utilizando faixa elástica, caneleiras de 1 a $2 \mathrm{~kg}$, jogos com bolas, exercícios rítmicos e de expressão corporal por meio de danças.

A fase de desaquecimento com exercícios de relaxamento muscular utilizando bolas com a técnica de distensionamento miofascial em braços, pernas, glúteos, coluna e pés, exercícios de expressão facial, automassagem facial e exercícios respiratórios 3 séries de 10 repetições.

\section{Análise dos Dados}

Foi utilizada análise descritiva para caracterização da amostra. Para análise estatística utilizou-se os softwares SPSS (Statistical Package for the Social Sciences) V20, Minitab 16 e Excel Office 2010. A normalidade das variáveis quantitativas de desfecho principal foi testada com o teste de Kolmogorov-Smirnov (KS) e definidos testes estatísticos paramétricos. Para testar e comparar os momentos pré e pós para todas as variáveis utilizou-se o Teste T-Student Pareado e para análise de correlação entre as variáveis foi utilizada a correlação de Pearson. Foi estabelecido Intervalo de Confiança (IC) para média de $95 \%$ e o nível de significância adotado foi de $p<0,05$.

\section{Resultados}

O Centro Dia no qual foi realizado o estudo é frequentado por 24 idosos. Nesse estudo, foram incluídos apenas 11 idosos, pois 13 não cumpriram os critérios de inclusão. 
Deste modo, os resultados deste estudo referem-se a 11 idosos, do sexo feminino, com média da idade de $88( \pm 4)$ anos; com nível de escolaridade Ensino Fundamental incompleto; frequência semanal no Centro-Dia com média de 4 $( \pm 1)$ vezes por semana e apresentaram uma pontuação média no MEEM de 12 ( \pm 5) representando uma perda cognitiva moderada. Estes foram submetidos a um total de 28 sessões de intervenção.

Todos os participantes apresentam pelo menos 2 alterações de saúde, além do déficit cognitivo, como hipertensão arterial, diabetes, limitação articular e esquizofrenia. Além disso, $81 \%$ da amostra possui plano de saúde. As características antropométricas que constituem a avaliação nutricional estão apresentadas na tabela 1.

Tabela 1. Características antropométricas do total amostra $(n=11)$

\begin{tabular}{lc}
\hline & Média/ Desvio Padrão \\
\hline Peso $(\mathrm{kg})$ & $53 \pm 9$ \\
Altura & $1 \pm 0$ \\
IMC $\left(\mathrm{Kg} / \mathrm{m}^{2}\right)$ & $24 \pm 4$ \\
Circunferência braço $(\mathrm{cm})$ & $26 \pm 3$ \\
Circunferência tríceps $(\mathrm{mm})$ & $16 \pm 4$ \\
Circunferência abdome $(\mathrm{cm})$ & $90 \pm 8$ \\
Circunferência cintura $(\mathrm{cm})$ & $77 \pm 14$ \\
Circunferência quadril $(\mathrm{cm})$ & $96 \pm 7$ \\
Circunferência panturrilha $(\mathrm{cm})$ & $32 \pm 4$ \\
\hline
\end{tabular}

Para verificar a diferença entre todas as variáveis avaliadas nos momentos pré e pós utilizou-se teste $T$-Student Pareado, segue dados na tabela 2.

Tabela 2. Comparação dos resultados alcançados em todos os testes no momento pré e pós intervenção $(n=11)$

\begin{tabular}{|c|c|c|c|c|c|c|c|c|c|}
\hline Momentos & & Média & Mediana & $\begin{array}{l}\text { Desvio } \\
\text { Padrão } \\
\end{array}$ & Min & Max & $\mathbf{N}$ & IC & P-valor \\
\hline \multirow{2}{*}{ Alcance sentado } & Pré & 22,3 & 20 & 10,4 & 10 & 47 & 11 & 6,2 & \multirow{2}{*}{$0,003^{*}$} \\
\hline & Pós & 28,2 & 26 & 9,0 & 16 & 48 & 11 & 5,3 & \\
\hline \multirow{2}{*}{ Alcance em pé } & Pré & 15,9 & 14 & 8,4 & 5 & 31 & 11 & 5,0 & \multirow{2}{*}{0,075} \\
\hline & Pós & 18,2 & 17 & 7,7 & 9 & 32 & 11 & 4,6 & \\
\hline \multirow{2}{*}{ Berg } & Pré & 45,2 & 49 & 9,2 & 21 & 53 & 11 & 5,4 & \multirow{2}{*}{0,274} \\
\hline & Pós & 42,3 & 45 & 12,4 & 14 & 53 & 11 & 7,4 & \\
\hline \multirow{2}{*}{ Caixa e blocos (Mão Direita) } & Pré & 56,7 & 60 & 13,2 & 35 & 78 & 11 & 7,8 & \multirow{2}{*}{0,425} \\
\hline & Pós & 54,5 & 60 & 17,8 & 22 & 80 & 11 & 10,5 & \\
\hline \multirow{2}{*}{$\begin{array}{c}\text { Caixa e blocos (Mão } \\
\text { Esquerda) }\end{array}$} & Pré & 52,8 & 54 & 10,2 & 34 & 64 & 11 & 6,0 & \multirow{2}{*}{0,347} \\
\hline & Pós & 50,3 & 51 & 16,6 & 20 & 73 & 11 & 9,8 & \\
\hline \multirow{2}{*}{ TUG } & Pré & 16,9 & 15 & 5,8 & 10 & 27 & 11 & 3,4 & \multirow{2}{*}{$0,009 *$} \\
\hline & Pós & 12,5 & 12 & 3,9 & 8 & 23 & 11 & 2,3 & \\
\hline
\end{tabular}

TUG (Timed up and Go); IC (Intervalo de Confiança); *valores estatisticamente significantes

Para verificar a correlação entre os testes foi realizada a Correlação de Pearson aonde foram encontradas correlações estatisticamente significantes como mostra na tabela 3. 
Tabela 3. Correlação dos Valores Médios alcançados no Pós teste $(n=11)$

\begin{tabular}{|c|c|c|c|c|c|c|}
\hline Pós & & $\begin{array}{l}\text { Alcance } \\
\text { sentado }\end{array}$ & $\begin{array}{l}\text { Alcance } \\
\text { em pé }\end{array}$ & Berg & $\begin{array}{l}\text { Caixa e } \\
\text { blocos } \\
\text { (Mão } \\
\text { Direita) }\end{array}$ & $\begin{array}{l}\text { Caixa e } \\
\text { blocos } \\
\text { (Mão } \\
\text { Esquerda) }\end{array}$ \\
\hline \multirow{2}{*}{ Alcance em pé } & Corr $(r)$ & $47,1 \%$ & & & & \\
\hline & P-valor & 0,143 & & & & \\
\hline \multirow{2}{*}{ Berg } & Corr (r) & $44,6 \%$ & $31,5 \%$ & & & \\
\hline & P-valor & 0,169 & 0,345 & & & \\
\hline \multirow{2}{*}{ Caixa e blocos (Mão Direita) } & Corr $(r)$ & $39,8 \%$ & $36,2 \%$ & $86,1 \%$ & & \\
\hline & P-valor & 0,225 & 0,274 & $0,001 *$ & & \\
\hline \multirow{2}{*}{ Caixa e blocos (Mão Esquerda) } & Corr $(r)$ & $46,4 \%$ & $36,1 \%$ & $80,8 \%$ & $96,4 \%$ & \\
\hline & P-valor & 0,151 & 0,276 & $0,003^{*}$ & $<0,001$ * & \\
\hline \multirow{2}{*}{ TUG } & Corr $(r)$ & $-53,2 \%$ & $-47,5 \%$ & $-68,4 \%$ & $-79,8 \%$ & $-80,2 \%$ \\
\hline & P-valor & 0,092 & 0,140 & $0,020^{*}$ & $0,003 *$ & $0,003 *$ \\
\hline
\end{tabular}

TUG (Timed up and Go); *valores estatisticamente significantes

\section{Discussão}

A interpretação dos resultados obtidos mostrou modificações em todas as variáveis avaliadas evidenciando que o protocolo de exercícios fisioterapêuticos proposto foi efetivo para saúde funcional dos idosos, no que diz respeito às funções e desempenho psicomotor.

A prevalência de idosos do sexo feminino neste estudo encontram apoio em dados da Organização Mundial da Saúde (OMS) e a Associação Internacional de Doenças de Alzheimer (AID). As organizações relatam que as mulheres idosas apresentam maior probabilidade de desenvolver demências. Tal fato pode ser atribuído a maior expectativa de vida nesse grupo e a procura por atendimento médico em maior proporção, quando comparado aos homens, o que contribui para o diagnóstico precoce das doenças ${ }^{14,15}$.

Nesse mesmo sentido, observa-se a prevalência de idosas longevas,e apresentando classificação média no MEEM representando uma perda cognitiva moderada. Diversos estudos relatam que a deterioração cognitiva é mais consistente e homogênea com avançar da idade acometendo mais a população feminina ${ }^{16}$. No entanto, a piora do quadro funcional varia muito e cada indivíduo pode apresentar diferente progressão, mas em geral o desempenho funcional é associado significativamente com a gravidade da demência ${ }^{17}$.
Os achados deste estudo apresentaram modificações em todas as variáveis avaliadas (tempo de realização da atividade ou escore total do teste), tanto na função psicomotora quanto no desempenho motor, embora sem significância estatística. Por exemplo, no Teste de Caixa e Blocos que avalia a destreza manual, notou-se diminuição no tempo médio de realização para mão direita e esquerda, igualmente no teste de alcance funcional em pé. Com exceção do equilíbrio estático e dinâmico avaliado pela Escala de Berg, no qual o escore diminuiu no pós, conforme ilustrado na tabela 2.

A simplicidade na execução da tarefa solicitado nos testes caixa e blocos e alcance funcional podem ter influenciado no resultado considerando o quadro demencial dos participantes. Em ambos os testes 0 comando era simples e exigia apenas um movimento, que estimulava a função psicomotora. Em contrapartida no teste de Berg as 14 tarefas propostas apresentam exigência motora associada à exigência cognitiva, como coordenação, equilíbrio estático e dinâmico e atenção, fatores que podem ter influenciado o resultado. Além disso, o protocolo de exercícios foi proposto considerando a idade, as condições de saúde, grau de independência e prevenção de quedas. De tal modo, que se optou por estimular a independência para as atividades de vida diária, como marcha em curtas distâncias, alimentação e autocuidado. Tal fato encontra apoio nos resultados para o teste de Alcance Funcional sentado e em pé, que também avalia controle motor de tronco e membros superiores (equilíbrio e mobilidade). 
Confirma-se em estudos prévios que o teste de alcance funcional apresentou aumento significativo pós treinamento com exercícios resistidos, equilíbrio, coordenação e alongamento $\stackrel{18}{\text {. E }}$ ainda que a prática de atividade física com uma frequência semanal de 2 sessões tem sido suficiente para melhoria e/ou manutenção da flexibilidade. Vale ressaltar que esse teste também pode avaliar o risco de queda, não apenas flexibilidade ${ }^{19}$.

De modo semelhante os achados para o TUG mostraram resultados significativos para mobilidade e diminuição do risco de quedas. Os achados nesse estudo encontram apoio em estudo de revisão onde os programas de exercícios físicos em idosos com DA mantém as funções cognitivas, com uma melhora da agilidade, do equilíbrio, sem aumento do risco de quedas 4 . A capacidade de atenção concentrada, a memória de curto prazo e o desempenho dos processos executivos, como planejamento de ações sequenciais logicamente estruturadas e capacidade de autocorreção das ações constituem funções cognitivas imprescindíveis na vida cotidiana. Realizar atividades com estímulos multissensoriais resulta em melhora principalmente na mobilidade funcional ${ }^{13}$.

Embora os resultados alcançados no teste de Berg não tenham sido satisfatórios nesse estudo. $\mathrm{Na}$ análise de correlação entre os resultados de Berg com TUG foi encontrada correlação negativa classificada como boa. Isso significa que as variáveis são inversamente proporcionais, ou seja, à medida que a pontuação do Berg aumenta, o valor do TUG em segundos diminui. Tal achado evidencia o efeito do protocolo realizado. A diferença encontrada no resultado do TUG pode ser compreendida, pois o teste exige deslocamento físico com mudanças posturais que estão diretamente relacionados à mobilidade trabalhada no protocolo de exercícios. Já os testes que compõe a escala Berg não foram amplamente abordados no protocolo, como por exemplo, equilíbrio dinâmico que depende da integração da sensação vestibular, responsável por mandar informações do movimento do corpo em relação à gravidade ao Sistema Nervoso Central, e periférica, visão, respostas neuromusculares como força muscular e tempo de reação ${ }^{20}$. Além disso, as participantes apesar de apresentarem DA classificada como moderada, apresentam independência ou semi-independência para atividades de vida diária e locomoção²1.
Outro achado relevante nesse estudo refere-se às correlações ilustradas na tabela 3. A correlação entre Berg e Caixa e blocos (Mão Direita) foi positiva de indicando que quanto maior o valor do Berg, maior será o valor de Caixa e blocos (Mão Direita), sendo assim diretamente proporcionais. Já entre o teste Caixa e blocos (Mão Direita) com TUG a correlação foi classificada como negativa, ou seja, inversamente proporcionais, indicando que quanto maior o valor alcançado no teste de Caixa e blocos (Mão Direita) menor será o valor do TUG. As duas condições foram classificadas como Ótima.

Embora a mão dominante das participantes do grupo seja a direita, a mesma condição foi encontrada para a correlação do Caixa e Blocos (mão esquerda) tanto com Berg quanto com TUG.

Há uma correlação entre a força manual, equilíbrio e marcha em idosos, quanto maior a força na mão melhor será o equilíbrio dinâmico e maior velocidade na marcha. Tal fato ocorre por existir correlação entre os valores medidos de força de preensão manual com a força muscular de membros superiores e inferiores. Para realização do teste de Caixa e Blocos é necessário força de preensão manual suficiente para que se possa realizar o teste com agilidade ${ }^{22}$.

Os resultados desse estudo revelaram que o protocolo proposto foi capaz de modificar a função psicomotora e de mobilidade em idosas com DA. Embora os resultados tenham sido satisfatórios vale ressaltar algumas dificuldades encontradas na execução do protocolo. Em virtude da condição cognitiva, alguns exercícios propostos foram executados com dificuldade pelas idosas, principalmente relacionados à compreensão da tarefa proposta e/ou limitação física derivada da condição cognitiva que não permitia a repetição do movimento. Como por exemplo, dificuldade com atenção; juízo; raciocínio; discurso; memória e imaginação

A vivência de exercícios em grupos tem uma mudança positiva por meio de reflexão e de estímulos decorrentes dos diálogos ou da observação ${ }^{23}$. Através da percepção dos próprios idosos constatou-se que a Fisioterapia trabalhada em grupo promove benefícios perante a limitação dos mesmos, facilitando execução dos movimentos durante os exercícios e além disso, facilita a interação, melhorando assim a relação social ${ }^{24}$. 
Outra limitação desse estudo refere-se à dificuldade de generalização dos dados pela falta de grupo controle, número reduzido de participantes incluídos e falta de homogeneidade em relação as condições de saúde.

Cientes de que os resultados alcançados não se configuram como irreversíveis, após a conclusão do estudo foi realizado orientação as cuidadoras do local e entrega de folhetos autoexplicativos como reforço contendo as sequências dos exercícios estabelecidos no protocolo para ser aplicado com as idosas no mínimo $2 x$ por semana.

\section{Conclusão}

O protocolo de exercícios fisioterapêuticos teve eficácia sobre a saúde funcional dos idosos com DA. Principalmente em relação à função psicomotora e o desempenho motor de alcance funcional e mobilidade, contribuindo significativamente para diminuir o risco de quedas e manutenção da capacidade funcional.

\section{Agradecimentos}

Agradecemos ao corpo docente do curso de Fisioterapia do Mackenzie, a Universidade Presbiteriana Mackenzie e aos colaboradores da AFAI.

\section{Contribuições dos autores}

Dias CQ participou da concepção, delineamento, coleta de dados, análise estatística dos dados da pesquisa, interpretação dos resultados e redação do artigo científico. João BSA participou da interpretação dos resultados e redação do artigo científico. Graciani, Z participou do delineamento e coleta de dados do estudo. Amato $\mathrm{CAH}$ da interpretação dos resultados e redação do artigo científico. Rodrigues E participou da concepção, delineamento, interpretação dos resultados. Vianna DL participou análise estatística dos dados e interpretação dos resultados. Fernandes SMS participou da concepção, delineamento, análise estatística dos dados da pesquisa, interpretação dos resultados e redação do artigo científico.

\section{Conflitos de interesses}

Nenhum conflito financeiro, legal ou político envolvendo terceiros (governo, empresas e fundações privadas, etc.) foi declarado para nenhum aspecto do trabalho submetido (incluindo, mas não se limitando a subvenções e financiamentos, participação em conselho consultivo, desenho de estudo, preparação de manuscrito, análise estatística, etc.).

\section{Referências}

1. Mendes TAB. Geriatria e Gerontologia - Série Manuais de Especialização. 1.ed. São Paulo: Manole; 2014.

2. Miranda GMD, Mendes ACG, Silva ALA. Population aging in Brazil: current and future social challenges and consequences. Rev Bras Geriatr Gerontol. 2016;19(3):507-19. doi: 10.1590/180998232016019.150140

3. Dawalibi NW, Anacleto GMC, Witter C, Goulart RMM, Aquino RC. Envelhecimento e qualidade de vida: análise da produção científica da SciELO. Estud Psicol. 2013;30(3):393-403. doi: 10.1590/S0103-166X2013000300009

4. Martelli A. Alterações Cerebrais e os Efeitos do Exercício Físico no Melhoramento Cognitivo dos Portadores da Doença de Alzheimer. Revista Saúde e Desenvolvimento Humano. 2013;1(1):49-60. doi: 10.18316/1021

5. Goyanna NF, Freitas CASL, Brito MCC, Mourão Netto JJ, Gomes DF. Idosos com doença de alzheimer: como vivem e percebem a atenção na estratégia saúde da família. Rev Fund Care Online. 2017;9(2):379-386. doi: 10.9789/2175-5361.2017.v9i2.379-386

6. Santos CS, Bessa TA, Xavier AJ. Fatores associados à demência em idosos. Cien Saude Colet. 2020;25(2):603-11. doi: $\underline{10.1590 / 1413-81232020252.02042018}$

7. Ferretti F. Efeitos de um programa de exercícios na mobilidade, equilíbrio e cognição de idosos com doença de Alzheimer. Fisioter Bras. 2016;15(2):119-25. doi: 10.33233/fb.v15i2.325

8. Melo DM, Barbosa AJG. O uso do Mini-Exame do Estado Mental em pesquisas com idosos no Brasil: uma revisão sistemática. Cien Saude Colet. 2015;20(12):3865-76. doi: 10.1590/1413$\underline{812320152012.06032015}$

9. Dias SMS, Silva RJM, Piazza L. Equilíbrio, mobilidade funcional e nível de atividade física de idosos institucionalizados que realizam e não realizam fisioterapia. ConScientiae Saúde. 2016;15(2):191-9. doi: $10.5585 /$ conssaude.v15n2.6253

10. Araújo DP, Barbosa PB, Franco CIF, Brito RG. Habilidade manual do idoso que vive com a família comparada com o idoso institucionalizado. Rev Neurociencias. 2010;18(4):448-53. doi: 10.34024/rnc.2010.v18.8430

11. Agner VFC, Gomes ARS, Paz LP, Correa CL. Identificação do perfil físico-funcional de idosos de uma instituição de longa permanência. Rev Pesqui Fisioter. 2013; 23;3(2):152-167. doi: $\underline{10.17267 / 2238-2704 r p f . v 3 i 2.159}$

12. Karuka AH, Silva JAMG, Navega MT. Analysis of agreement of assessment tools of body balance in the elderly. Rev Bras Fisioter. 2011;15(6):460-466. doi: 10.1590/S1413-35552011000600006 
13. Cascon RM, Brown A, Dias I, Cardozo D, Salerno VP, Simão R. Efeito do treinamento de força na potência muscular de membros inferiores de idosos coronariopata. ConScientiae Saúde. 2017;16(1):26-32. doi: 10.5585/conssaude.v16n1.6881

14. Fernandes MRS, Melo MST, Maranhão TLG, Sampaio FTP, Batista HMT. Doença de Alzheimer nas Mulheres: Prejuízos Pessoais e Luto Familiar. Id On Line Rev Psic. 2018;12(39):533-51. doi: 10.14295/idonline.v12i39.1012

15. Lira M, Santos LCCS. Correlação entre função cognitiva e capacidade funcional nos indivíduos com doença de Alzheimer. Cad Pós-Graduação em Distúrbios do Desenvolv. 2012;12(2):3645.

16. Parmera JB, Nitrini R. Demências: da investigação ao diagnóstico. Rev Med. 2015;94(3):179-84. doi: 10.11606/issn.16799836.v94i3p179-184

17. Talmelli LFS, Vale FAC, Gratão ACM, Kusumota L, Rodrigues RAP. Doença de Alzheimer: declínio funcional e estágio da demência. Acta Paul Enferm. 2013;26(3):128-39. doi: 10.1590/ S0103-21002013000300003

18. Souza LA, Fernandes AB, Patrizzi LJ, Walsh IAP, Shimano SGN. Efeitos de um treino multissensorial supervisionado por seis semanas no equilíbrio e na qualidade de vida de idosos. Med. 2016;49(3):223-31. doi: 10.11606/issn.2176-7262.v49i3p223-231
19. Beserra LRS. Análise da flexibilidade em idosos participantes de atividades físicas no SCFV Serraria-PB [dissertação]. Borborema (PB): Universidade de Brasília; 2014.

20. Ferretti F, Beskow GCT, Slaviero RC, Ribeiro CG. Análise da qualidade de vida em idosos praticantes e não praticantes de exercício físico regular. Estud Interdiscipl Envelhec. 2015;20(3):729-743.

21. Ruzene JRS, Navega MT. Avaliação do equilíbrio, mobilidade e flexibilidade em idosas ativas e sedentárias. Rev Bras Geriatr Gerontol. 2014;17(4):785-93. doi: 10.1590/1809-9823.2014.13105

22. Brito AO. Correlação entre a força de preensão manual com teste de alcance funcional e o teste timed up and go [dissertação]. Brasília: Universidade Católica de Brasilia; 2015.

23. Machado WD, Gomes DF, Freitas CASL, Brito MCC, Moreira ACA. Idosos com doenças crônicas não transmissíveis: um estudo em grupos de convivência. ReonFacema. 2017;3(2):445-51.

24. Menezes JNR, Costa FJS, Lima AKR, Souza CGD, Oliveira L, Santos RC. Atividades fisioterapêuticas em grupos para idosos institucionalizados: a percepção do idoso. Rev FisiSenectus. 2018;5(2):47. doi: 10.22298/rfs.2017.v5.n2.4124 